\title{
MUJER, POLÍTICA Y MÚSICA: DOS TEXTOS OLVIDADOS DE YOLANDA OREAMUNO
}

\author{
Alexánder Sánchez Mora
}

\begin{abstract}
RESUMEN
El hallazgo del ensayo ¿Puede la mujer tener los mismos derechos políticos que el hombre? (1932) permite retrotraer la fecha inicial de la actividad literaria de Yolanda Oreamuno y ofrece una idea de su proceso de maduración intelectual. La crónica Su música (1937), publicada por el vespertino La Hora, permite acercarse a un momento de fuerte compromiso político de la joven escritora. Palabras clave: Yolanda Oreamuno, literatura costarricense, ensayo, crónica, música, política.
\end{abstract}

\begin{abstract}
The discovery of the essay ¿Puede la mujer tener los mismos derechos politicos que el hombre? (1932) pushes back the initial date of the literary activity of Yolanda Oreamuno and provides an understanding of the process of her intellectual maturation. The chronicle, Su música (1937), published by the journal La Hora, allows one to better understand the strong political commitment of the young writer.

Key words: Yolanda Oreamuno, Costa Rican Literature, essay, chronicle, music, politics.
\end{abstract}

Uno de los elementos constitutivos del aura mítica ${ }^{1}$ de Yolanda Oreamuno es el misterioso paradero de sus textos, que ha provocado una multitud de especulaciones y búsquedas, en su mayor parte infructuosas. Una sola novela publicada - la muy justamente elogiada La ruta de su evasión (1948) - y otras cinco inéditas -Por tierra firme (1936), Dos tormentas y una aurora (¿1944?), Casta sombría (1944), De hoy en adelante (1947) y José de la Cruz recoge su muerte (¿?) (Vallbona 1995: 22-23)-, algunas de las cuales no se sabe si las escribió o solamente las soñó, al decir de García Carrillo, apenas conocidas por comentarios de la escritora o referencias

\footnotetext{
Alexánder Sánchez Mora. Profesor de la Escuela de Filología, Lingüística y Literatura de la Universidad de Costa Rica. San Pedro, San José, Costa Rica.

Correo electrónico: alsanchezm@hotmail.com

Recepción: 17- 9- 2008

Aceptación: 23- 10- 2008
} 
de lectores privilegiados, han contribuido a hacer de la fijación del corpus literario de Yolanda Oreamuno uno de los mayores retos de la historiografía literaria costarricense.

La suerte de los textos menores -relatos y escritos periodísticos- de Oreamuno también ha sido incierta. Una primera recopilación, A lo largo del corto camino (1961), realizada por Lilia Ramos, Enrique Macaya y Julián Marchena, reunió sus publicaciones en el Repertorio Americano. Con posterioridad, los trabajos de Rima de Vallbona, Yolanda Oreamuno (1971) y La narrativa de Yolanda Oreamuno (1995), dieron cuenta de nueve textos olvidados, ocho de ellos publicados en Guatemala y México y otro inédito. Sin embargo, es sabido que aún no han sido localizados algunos de los relatos que, en 1961, Lilia Ramos calificaba como perdidos: "El lobo en el aprisco", "Las bodas de Canaan" y "La tía tenía trenzas" (Ramos 1961: 336).

Hasta el momento, se creía que los primeros textos de Oreamuno eran "Para Revenar, no para Max Jiménez" y "La lagartija de la panza blanca", ambos publicados en el Repertorio Americano en 1936 (Oreamuno 1961). Otra conjetura, con muchos visos de probabilidad, es que tal condición le corresponde al ensayo "Medios que usted sugiere al colegio para librar a la mujer costarricense de la frivolidad ambiente", que apareció en el Repertorio Americano en $1938^{2}$. Rima de Vallbona estima que este texto, con el que Oreamuno obtuvo una mención de honor en un concurso literario del Colegio Superior de Señoritas celebrado en 1933, fue escrito cuando la escritora contaba entre los dieciséis y los diecisiete años (1971: 55).

Dadas tales fechas, se puede afirmar que el "descubrimiento” del ensayo "¿Puede la mujer tener los mismos derechos políticos que el hombre?", de 1932, permite retrotraer la fecha inicial de la actividad literaria de Yolanda Oreamuno. En efecto, en ese año, cuando era una estudiante de dieciséis años de edad, la novel escritora participa en un concurso en el Colegio Superior de Señoritas y comparte el primer premio con Cristina Thompson Q. Ambos ensayos son publicados en la Revista Costarricense el 16 de octubre de 1932.

Este primer artículo conocido de la muy joven escritora puede resultar de mucho interés para los conocedores de su producción posterior. Ante el tema de los derechos políticos de la mujer, por entonces muy en boga merced a los esfuerzos de la Liga Feminista-fundada en 1923-, Oreamuno responde con una adscripción total al discurso conservador y patriarcal que niega la conveniencia de que el ejercicio de tales derechos no haga distinciones de género. La posición de Oreamuno resulta más llamativa si se considera que se enuncia dentro de una institución, como el Colegio Superior de Señoritas, que desempeñó un destacado papel en la difusión de las ideas de las sufragistas costarricenses. Prueba de ello es que fue un grupo de estudiantes de esa institución el que, en escrito del 20 de junio de 1923, presentó ante el Congreso la primera solicitud para que se aprobara el voto femenino. Ese mismo año, se funda la Liga Feminista durante un acto celebrado en el mismo colegio y su directora, Esther de Mezzerville, fue designada Vicepresidenta (Barahona Riera 1994: 74-77) ${ }^{3}$. Durante los siguientes años, la Liga desplegaría una intensa actividad en procura de obtener la aprobación legislativa para el sufragio de las mujeres. Para hacer más patente el carácter contracorriente de Oreamuno, su breve ensayo es publicado por la Revista Costarricense precedido por una nota, presumiblemente escrita por Sara Casal, directora de la revista y una de las más importantes dirigentes feministas, en la que se critica la negativa de la colegiala a apoyar el sufragio femenino.

Macaya afirma que es a partir de 1938 cuando Yolanda Oreamuno inicia la búsqueda de una autoafirmación femenina que se distancie de la mirada masculina, ajena y engañosa, y que se fundamente en "una definición de la mujer surgida desde ella misma" (1997: 36). No sería por ello sorprendente que en 1932 aún no se hubiera deslindado del discurso patriarcal 
que proyecta a la mujer ideal como un ser que debe mantenerse al margen de la esfera pública. Tal y como señala Aldaraca: "The ideal woman is ultimately defined not ontologically, not functionally but territorialy, by the space wich she occupies. The frontier of her existence as a virtuous woman begins and ends at her doorstep" (citada en Hoffman 2004: 43). El texto primerizo de Yolanda Oreamuno muestra, por lo tanto, una etapa temprana, poco o nada considerada hasta ahora, de todo un proceso de reflexión que la llevará a cuestionar los fundamentos mismos de la dominación masculina.

Cinco años después, el 5 de noviembre de 1937, el vespertino La Hora, notable y polémico periódico dirigido por el escritor José Marín Cañas, publicó la crónica Su música, en la que Oreamuno reseña el concierto del pianista argentino Óscar Luis Karzag ${ }^{4}$ y la impresión que le produjo su música descriptiva. Este texto, aun más que el anterior, pasó desapercibido y a ello contribuyeron los acontecimientos en que se vio envuelta la escritora. Lo que sucedió a continuación da cuenta del proceso de construcción de la imagen legendaria de Yolanda Oreamuno.

En la edición de ese mismo día, un titular de la portada de La Hora informa de la destitución de Yolanda Oreamuno de su puesto en Tributación Directa por haber participado en el "sabotaje" de la presentación de González Marín, un declamador franquista, en el Teatro Raventós. La nota periodística lleva por título "Fue destituida la Sra. Yolanda Oreamuno", aunque en realidad el despido laboral de Oreamuno es apenas una consecuencia marginal de los sucesos que allí se relatan. De hecho, el texto se centra en la descripción detallada de la forma en la que los miembros de la Liga Antifacista -entre ellos, Francisco Marín Cañas, hermano del director de La Hora - y algunos comunistas impidieron la realización del acto del declamador español y del posterior enfrentamiento de los manifestantes con la policía. El título de la nota, en consecuencia, resulta engañoso y cumple una función distractora, por cuanto desplaza la atención del lector de los hechos políticos (el boicot de un acto organizado por los simpatizantes rebeldes franquistas) hacia la participación de una mujer notable por su belleza (un par de años atrás, Oreamuno había sido la candidata de La Hora en un publicitado certamen de belleza). O bien, por el contrario, atrae la atención del público hacia una información que de otra manera habría pasado desapercibida y, de paso, vende la noticia gracias al atractivo de una mujer ya por entonces famosa en el reducido espacio social josefino.

En un artículo publicado el 13 de noviembre de 1937 en Trabajo, la dirigente comunista Carmen Lyra ofrece una visión de los mismos hechos con un énfasis muy diverso. Su texto denuncia que un grupo de "mujeres trabajadoras antifascistas", entre las que destaca a Luisa González, Clemencia Valerín e Isabel Salazar, fueron detenidas durante los disturbios en el Teatro Raventós: “PPor qué se metieron Luisa González, Clemencia Valerín, Isabel Salazar y demás compañeras a protestar por el recital de González Marín y la barbarie con que el Coronel Gallegos protegió a los fascistas de Costa Rica?” (1937: 424).

En la versión de Carmen Lyra, se destaca la participación en la protesta de mujeres de extracción obrera y comprometidas en forma directa con el Partido Comunista, mientras que ni siquiera se menciona el incidente protagonizado por Yolanda Oreamuno. Más de sesenta años después de los disturbios del Teatro Raventós, el destacado escritor comunista Joaquín Gutiérrez, quien en 1937 contaba con diecinueve años de edad, rememoró la participación de Yolanda Oreamuno:

El plan era dejar al recitador González Marín que entrara contoneándose, al abrirse las cortinas para iniciar el acto, dijera el primer poema y, apenas dijera el título del segundo, descargar nuestras municiones. Mientras tanto, Yolanda se levantaba de su asiento, y, haciéndose la que iba a fumar, apagaba la luz general (Gutiérrez 2000: 171). 
La versión de Gutiérrez se distancia en mucho de la consignada por los periódicos que dieron cuenta de los acontecimientos, La Hora y La Tribuna, lo cual puede atribuirse tanto a la gran distancia temporal entre los incidentes (1937) y el momento de la escritura del texto de Gutiérrez (1999), como al carácter mismo del escrito autobiográfico en el que los hechos históricos son sometidos a un tratamiento literario. Lo cierto es que esta reconstrucción asigna a Oreamuno un papel protagónico muy diverso del que en efecto cumplió; de la información periodística se concluye que Oreamuno nunca llegó a apagar las luces del teatro, tal y como lo afirma Gutiérrez, sino que se limitó a increpar al declamador con un enérgico "Cállese"5. Este parece ser un caso más en el que la leyenda de Yolanda Oreamuno supera y da forma a la realidad sobre su vida 6 .

Estos dos artículos marcan otros tantos momentos, más allá de lo literario strictu sensu, que parecieran mostrar una flagrante contradicción: el primero, una decidida negativa a aceptar la participación femenina en los asuntos políticos, y el segundo, tan solo cinco años después, publicado en una coyuntura en la que la escritora da muestras de un compromiso político que va más allá de la retórica y que afirma, con los hechos, la condición impostergable de la mujer como protagonista de la vida política. Una muestra de tal activismo fue plasmada por el embajador estadounidense en San José, Robert M. Scotten, en un informe fechado el 23 de julio de 1942:

\footnotetext{
Yolanda Oreamuno de Barahona is the wife of Oscar BARAHONA Streber, an able young lawyer who holds an important post in the Ministry of Salubridad Pública. She is a very handsome young woman and was formerly the wife of a Chilean Chargé d' Affaires here. She has frequently taken part in demonstrations for Russia and against the Axis and Falangist Spaniards and has even been committed to jail for excess of zeal ${ }^{7}$.
}

En 1932 y en 1937, ya fuera como alumna de Primero de Comercial del Colegio Superior de Señoritas o como funcionaria de Tributación Directa, ya fuera conservadora o liberal, Yolanda Oreamuno se define como una mujer marcada por la contradicción frente al poder: así, en un momento se muestra patriarcal en un ambiente dominado por el feminismo y, en otro, republicana ante un gobierno filofranquista. Y en ambas situaciones debe sufrir las consecuencias - la descalificación de su ensayo mediante la nota introductoria de Sara Casal en un caso y la pérdida de su empleo en el otro-de sus gestos retadores.

\section{Notas}

1. "Yo considero que el mito de Yolanda Oreamuno no existe: lo que existe, perenne y renovado siempre, es su obra, indisoluble de su quehacer literario pero inmersa también en su tragedia personal, colectiva y generacional, en donde ella se buscó varias patrias para renegar amorosamente de la suya..." (Chase 1977: 38).

2. En diversos estudios, por ejemplo en Macaya (1997) y Vallbona (2006), se ha atribuido a este ensayo el título de “¿Qué hora es?”, lo cual es una confusión pues ese era el nombre de la sección del Repertorio Americano en la que fue publicado.

3. Sobre las luchas por el sufragio femenino en Costa Rica, véase Rodríguez Sáenz (2002) y Solano Arias (2005). Herrera Zavaleta (2002: 136-138) discute la actitud refractaria de las militantes del Partido Comunista a acercarse al movimiento sufragista, al que consideraban "liderado por mujeres de "clase alta' y anticomunistas". Molina Jiménez (2002) señala algunas posibles causas del distanciamiento de la más importante intelectual comunista, Carmen Lyra, respecto de las propuestas sufragistas. 
4. A pesar de una intensa búsqueda, incluso con la ayuda de asociaciones musicales argentinas, no ha sido posible obtener información alguna sobre Óscar Luis Karzag.

5. "Cuando el recitador González Marín iniciaba uno de sus versos, la señora Yolanda Oreamuno protestó en voz alta, diciéndole: "Cállese", lo que originó que fuera invitada a abandonar la sala". La Hora, 5 de noviembre de 1937, p. 6.

6. Joaquín Gutiérrez dedica un capítulo de sus memorias a la figura de Yolanda Oreamuno. Allí relata algunos de los episodios más novelescos de su vida: el disparo que hizo contra un grupo de jóvenes voyeuristas, su frustrado rapto, su fugaz matrimonio con un diplomático chileno y los asedios románticos de que fue objeto por parte de varios destacados artistas (2000: 155-160).

7. Agradezco a Iván Molina por esta información. Para un análisis detallado de la imagen de la política costarricense que construyeron los diplomáticos estadounidenses de la época, véase Molina Jiménez (2007).

8. El ensayo de Oreamuno fue precedido por la siguiente nota: "REVISTA COSTARRICENSE se complace en publicar los dos trabajos que obtuvieron el premio en el certamen de ortografía que se verificó en el Colegio de Señoritas. Ambos son muy hermosos trabajos y felicitamos a las dos señoritas vencedoras. Profundamente feministas en el sentido de igualdad de derechos políticos, no estamos de acuerdo con la idea sustentada por la señorita Oreamuno Unger, de que a la mujer no debe interesarle la política; no obstante, lo publicamos porque uno de los ideales de esta Revista es interesar a la mujer en el desarrollo de las ideas, y aplaudir siempre que se trate de elevar el nivel moral y cultural de la mujer".

\section{Bibliografía}

Barahona Riera, Macarena. 1994. Las sufragistas de Costa Rica. San José: Editorial de la Universidad de Costa Rica.

Chase, Alfonso. 1977. “Introducción”. En: Oreamuno, Yolanda. Relatos escogidos. San José: Editorial Costa Rica.

Gutiérrez, Joaquín. 2000. Los azules días. San José: Editorial de la Universidad de Costa Rica.

Herrera Zavaleta, Rosalila. 2002. "Maestras y militancia comunista en la Costa Rica de los años treinta”. En: Rodríguez Sáenz, Eugenia (ed.). Un siglo de luchas femeninas en América Latina. San José: Editorial de la Universidad de Costa Rica, 131-146.

Hoffman, Joan. 2004. “'¡Si no fuese por el decoro!’: Emilia Pardo Bazán’s Working Girls and the Polite Fiction of the Domestic Ideal". Hispanófila. 142: 43-54.

Lyra, Carmen. 1937. "Alrededor de los ecos antifascistas del recital de González Marín. El antifascismo de nuestras compañeras es combativo". En: González Gutiérrez, Luisa. 2006. Escritos. Heredia: EUNA, 423-425. 
Macaya, Emilia. 1997. Espíritu en carne altiva. San José: Editorial de la Universidad de Costa Rica.

Molina Jiménez, Iván. 2000. “Introducción”. En: Lyra, Carmen y Fallas, Carlos Luis. Ensayos políticos. San José: Editorial de la Universidad de Costa Rica.

2007. Anticomunismo reformista. San José: Editorial Costa Rica.

Oreamuno, Yolanda. 1961. A lo largo del corto camino. San José: Editorial Costa Rica.

Ramos, Lilia. 1961. "Yolanda Oreamuno en mi recuerdo eviterno". En: Oreamuno, Yolanda. A lo largo del corto camino. San José: Editorial Costa Rica, 331-342.

Rodríguez Sáenz, Eugenia. 2002. "La lucha por el sufragio femenino en Costa Rica (18901940)”. Rodríguez Sáenz, Eugenia (ed.). Un siglo de luchas femeninas en América Latina. San José: Editorial de la Universidad de Costa Rica, 87-110.

Solano Arias, Marta Eugenia. 2005. La Liga Feminista Costarricense y el inicio del largo camino por el voto de las mujeres en Costa Rica. Tesis de Maestría: Universidad de Costa Rica.

Vallbona, Rima de. 1971. Yolanda Oreamuno. San José: Ministerio de Cultura, Juventud y Deportes.

1995. La narrativa de Yolanda Oreamuno. San José: Editorial Costa Rica.

\section{ANEXOS}

¿Puede la mujer tener los mismos derechos políticos que el hombre?

(Composiciones premiadas en el Concurso de Redacción celebrado en el Colegio Superior de Señoritas) ${ }^{8}$

Desde el día en que Dios hizo la creación, estableció ciertos planos lógicos y legales, que colocan a cada ser en un sitio diferente del cual no puede salir por la ley natural de los hechos.

La mujer, a pesar del desenvolvimiento a que la ha autorizado la civilización, no puede salir de este plano. Sin embargo, nosotras las mujeres, podemos, queremos y debemos extender nuestra influencia a todo lo que nos rodea. La mujer de hoy, no es ya la que sólo borda y cuida a sus hijos, la que ríe en su casa; nó, es un elemento de este núcleo que avanza siempre hacia el más allá; la mujer ha adquirido derechos que antes no tenía, pero sobre todo y por todo, debe seguir siendo, la mujer.

Los hombres le piden hoy, su compañerismo, su impulso, su adhesión, pero también le piden su feminidad. 
El día en que la mujer esté a la par del hombre en el plano político, habrá dejado de ser ella para ser él.

Nosotras tenemos un derrotero que debemos agrandar, depurar y seguir, pero si queremos abarcar bajo nuestro poder, los que hasta hoy han sido derechos del hombre, no podremos cumplir con nuestra misión primordial, la de educar.

Si queremos ser grandes, si queremos tocar la gloria, abarcar el poder, qué gloria más grande, qué poder más sublime que el de modelar almas?

La política no se ha hecho para la mujer; es ilógico pretender ser jueces y partes en este dilema.

La mujer que quiera sentarse en las sillas del Congreso, la que quiera vivir esa vida agitada y pujante de la política, que selle las puertas de su casa y anule su personalidad.

La mujer no puede, ni debe tener los mismos derechos políticos del hombre.

\section{Yolanda Oreamuno Unger}

\section{Comercial}

Revista Costarricense, n. 75,16 de octubre de 1932, p. 1188.

\section{Su música}

El profesor Luis Óscar Karzag nos dio su música antenoche. Una música extraña y desconcertante. Ha costado mucho hacerlo tocar. Es vanidoso y exigente. Pero me siento compensada. Es más, todavía después que vacié aquí lo que esa música me dejó adentro, siento que le quedaré debiendo.

Se sale con la suya.

Para empezar. Casi no sé con qué empezó, porque hubo una cosa que quedó vibrando y que sostuvo a través de los valses, -que no me gustan- y a través de las variaciones arpegiales y de todos sus alardes de ejecución, es su música descriptiva.

De eso es que quiero hablar. la Hilandera, El Payaso, Amanecer en la Selva y sobre todas, La Tempestad.

No sabré nunca cómo está tan brutalmente conseguido el movimiento del trabajo maquinal de la hilandera sosteniendo dolorosamente la red de sus sueños de la mujer atada a su máquina; cómo hace saltar en su mano izquierda audazmente al payaso, que después de todo muere como todos los payasos, haciendo reír y levantando con su última farsa la carcajada final, cómo trabaja nota a nota, con deleite y con seguridad el despertar de los bichos todos en la selva y el juego del sol sobre las hojas, todo, sin obligar a nadie a derretirse por dentro en emociones sentimentaloides, y dando completa, la impresión de ver y de coger casi, el colorido brillante de todas estas cosas.

Pero en La Tempestad quedan cuajadas de golpe y a la definitiva las impresiones todavía un poco dispersas.

Karzag, con un poco de prestidigitador como alguien dijo, hace apagar la luz. Es mejor. Así no nos veremos las caras de espanto, de desconcierto, de suspensión.

Y empieza la tempestad. El mar se estira bajo las manos obedientes del hombre, porque ya no es la nota dócil a la mano, sino el hombre obediente a sonido y como una simple fórmula de él. 
Y el trueno vibra abajo en las notas graves. Karzag dice que esto no tiene pedales. Ya eso no importa porque las olas se levantan brutales una luego de la otra rajadas por el relámpago que oímos, digo oímos, dando un salto nervioso y defensivo. Y hay olitas chiquitas y crestas blancas y curvas ondas en el agua tumultuosa que oímos también. La tempestad se abre sobre el piano, retumba dura allá dentro en la caja sonora, brincan las olitas chiquitas zafando el cuerpo y todo crece desmesuradamente, el oleaje, el trueno, el crujido y el rayo.

Ya casi no se puede aguantar más. Se ahoga uno, busca inútilmente agarrarse a algo, ya todo ha llegado al punto donde el razonamiento más sereno y frío no puede pensar en notas, ni en teclas, ni en manos. Hay que salvarse a toda costa. Yo, me asfixio lento.

Dichosamente, sí, dichosamente, esto acaba. Se retira sobre bemoles la crecida y el mar se abre de pecho. Los truenos siguen sonando cada vez más lejanos y las olas relajadas, vuelven a bajar al teclado blando. Ya era tiempo. Respiramos con satisfacción y nos sentimos definitivos en tierra firme otra vez.

Luis Óscar Karzag tiene genio, pero tiene talento también. Si nos hubiera dejado allí, la angustiosa sensación hubiera perdurado. Hay que sacarnos del idiotismo completo.

Allí está la música india. Sí. Música india legítima, motivos que ha tomado entre los indios del Brasil, Ecuador, Bolivia y Perú. No está mixtificado el Yaraví ni el Himno al Sol tiene ritmo de fox, está armonizado con respeto y devoción, conservando formas musicales y melodías dentro de la fórmula que él pone.

La ilustra explicando con su español vocalizado en sajón, tomando de su personalidad agresiva y de su vida aventurera y extraña.

Es deliciosa y simple; es lo que necesitábamos después de La Tempestad.

Luis Óscar Karzag me ha dejado clara esta concepción.

La música descriptiva consigue más que el cuadro.

El movimiento que es esfuerzo mental en el cuadro, es dejarse llevar en el sonido. Al mirar un cuadro, tenemos obligadamente que crear un punto de comparación para saber como es el cuadro; este punto de comparación es la idea que de él nos formamos en la imaginación.

Si la pintura coincide con esa imagen, nos gusta, si nó no. Y luego hay que trabajar para movilizar esa concepción.

En la música descriptiva toda esa labor huelga. Las cosas se ven. Imaginativamente, pero libres de la forzada comparación. Y el resto del esfuerzo, el esfuerzo motor, lo hace el sonido. No hay más que dejarse ir. Es más viva y más local que la pintura.

Gracias Luis Óscar Karzag, la noche ha sido deliciosa y la lección vasta. Muchas gracias.

\section{Yolanda OREAMUNO}

La Hora, 5 de noviembre de 1937, p. 3. 